\title{
Validity for Strong Pluralists
}

\author{
Aaron J. Cotnoir ${ }^{\dagger}$ \\ Northern Institute of Philosophy \\ University of Aberdeen
}

Truth pluralists accept that there are many truth properties. But truth pluralists disagree over whether any of these truth properties apply generally across the whole class of propositions, or whether their application is in some way restricted. Global truth pluralists accept that all truth properties are general. Adherents (e.g. Beall [6], Field [17], Greenough [18], McGee [27, 28], Scharp [33, 34]) have usually been led to pluralism by way of considering the semantic paradoxes. Weak truth pluralists accept that there is at least one general truth property. The weak pluralist approach has been most vigorously defended by Michael Lynch [26]. Other adherents include Dorsey [12, 13], Edwards [16], Kölbel [21], Pedersen [31], and Sher [37, 38]. Finally, I defend strong pluralism, the view that does not accept a general truth property. Crispin Wright $[42,43]$ - who accepts the existence of a single general and univocal truth concept but rejects the existence of any property associated with it - appears to be the only other adherent ${ }^{1}$

I think that strong pluralism has not recieved sufficient attention. Perhaps this is due in large part to strong pluralists' difficulties in solving some formal problems faced by truth pluralists, such as Tappolet's [39] Problem of Mixed Inferences.2. In this paper, I will argue that the strong pluralist's prospects for solving this problem are not as hopeless as it might first appear; the Problem of Mixed Inferences can be solved 3

In $\S 1$, the problem is presented, and attempted solutions in the literature consistent with

\footnotetext{
${ }^{\dagger}$ Penultimate DRAfT: please cite the published version (forthcoming in Philosophy and Phenomenological Research).

${ }^{1}$ But see his most recent [44], where he tentatively accepts a 'transparent' or 'abundant' general truth property. Pedersen and Wright [29] have expressed views that are strongly pluralist in spirit.

${ }^{2}$ There are other closely related problems. The Problem of Mixed Compounds is sometimes credited to Tappolet [40], but was first put forward by Williamson [41]. See also Edwards [14, 15] for further discussion. Even less attention has been paid to a particularly difficult variant: the Problem of Mixed Generalizations, which as far as I can tell was first put forward by Lynch [23]. See Dorsey [12] for a variant of Lynch's [26] solution, and Shapiro [35] for further discussion. These problems might be thought to originate from a more general issue involving ambiguity, as was first suggested by Williamson [41].

3Of course, a full defense of strong pluralism will have to address the Problem of Mixed Compounds and the Problem of Mixed Generalizations as well. I have given a viable solution to the former in [10]. I address the latter problem (and other difficulties surrounding quantification) in [9]. Further motivation for strong pluralism involving the semantic paradoxes is given in my [11].
} 
strong pluralism are summarized. In §2.1, I give a formal semantics for strong pluralists utilizing an algebraic definition of validity. $\$ 2.2$ shows that this semantics yields the consequence relation for classical propositional logic. In §2.3, I argue that the algebraic definition solves the problem to Tappolet's specifications. In §2.4, I respond to some initial objections. §3 follows suggestions by Lynch [26] and Wright [42] by extending this approach to non-classical domains, showing how the account can handle domains for which paracomplete, paraconsistent, or intuitionistic logic may be most appropriate. The result, I claim, is a standard semantic account of validity that can do all the work the pluralist requires, without appeal to any general truth property.

\section{The Problem of Mixed Inferences}

Most pluralists who accept the existence of less-than-general truth properties have followed Wright [42] (and later Lynch [23]) in restricting those properties to 'domains' or 'regions of discourse' or 'areas of inquiry' 4 Roughly speaking, domains are intended to correspond to different subject matters: science, mathematics, history, morality, religion, aesthetics, etc. Pretheoretically, there appear to be reasons for thinking that truth-in-science is different from truth-in-morality which is different from truth-in-mathematics. Domain-based pluralists take these appearances seriously, and attempt to work out a rigorous theory on this basis. Viewed in this way, the project of individuating domains depends on the possibility of individuating subject matters.5 The task of carrying out this project is a difficult and important one, but its results are largely independent of the arguments of this paper ${ }^{6}$ For the purposes of this paper, I take domains to be subsets of the class of propositions. It is assumed that these subsets are

\footnotetext{
${ }^{4}$ There are a few notable exceptions. Barnard [4, 3] and Horgan [19] restrict truth properties to contexts. Sher's [37] view too does not relativize truth to domains, but rather to 'factors' or criteria of application. However, Sher may not be so far from Wright on this issue. She writes, "A pluralism based on factors of truth is finer and more nuanced than one based on fields [domains] of truth, yet it recognizes (and can account for) differences among truths in difference areas of discourse." (21)

5 Gila Sher $[38$ and Cory Wright $[46]$ attempt to translate these difficulties into an objection to domain-based pluralism. Claire Horisk and Stewart have recently pressed this point in discussion.

${ }^{6}$ The difficulties arise most transparently in the context of predicate logic, where objects and properties come to the fore. Typically, domains are thought to be individuated by a taxonomy on concepts or predicates (cf. Lynch [24] p. 400). But vagueness inherent in most concepts/predicates complicates matters. In [9], I suggest a new approach to domain individuation based not on concepts/predicates, but on objects. This approach avoids some problems of the earlier approaches.
} 
disjoint - no proposition is a member more than one domain; although this assumption is by no means necessary in what follows. Because the approach to validity given below is consistent with a wide variety of views about the nature and number of domains, these issues are left for further exploration elsewhere.

Suppose there is a domain for moral discourse, and a domain for descriptive discourse. Presumably, we can make the following inference:

1. Torture is wrong.

2. The United States tortured prisoners at the Abu Ghraib prison.

3. So, the United States did something wrong.

Now, (1) seems morally true. And (2) is descriptively true. Can we then validly infer the (morally true, but not descriptively true) conclusion?

Tappolet [39] writes:

The validity of an inference requires that the truth of the premises necessitates the truth of the conclusion. [...] For the conclusion to hold, some unique truth predicate must apply to all three sentences. But what truth predicate is that? And if there is such a truth predicate, why isn't it the only one we need? (209)

These questions are intended as a problem for truth pluralists generally 7 Tappolet sees the problem as trilemma: either (a) reject that any mixed inferences are valid, (b) accept a general property that is preserved over mixed inferences, or (c) reject the standard characterization of validity as necessary truth preservation. Tappolet regards option (a) as unpalatable given the existence of mixed inferences like the example above. In response to option (b), Tappolet worries that this general property is to be thought of as a truth property. And as such, Tappolet is here giving voice to what some have called the 'instability challenge' ${ }^{8}$ But the instability challenge is largely a problem for weak pluralists; and so I leave debate over horn

\footnotetext{
7Tappolet puts the problem in terms of truth predicates, but can easiliy be translated into talk of truth properties. For more detail on the property/predicate distinction and how it relates to truth pluralism, see [30].

${ }^{8}$ See Pedersen [31] for a description of the challenge, and lines of response. See also Cory Wright [45].
} 
(b) to them.9 Option (c) is thought to be a last resort; Tappolet suggests that one ought to reject truth pluralism before revising the concept of validity.

At first glance, however, the third horn of the trilemma does not appear to be too sharp. A proof-theoretic characterization of validity seems an obvious non-revisionary suggestion. Tappolet [40] considers this response.

The distinction between [kinds of] truth does not appear to allow for any difference in the inferential role of propositions. So there would be no formal difference between the two kinds of truth. The kind of truth involved in the disjunction introduction rule, for instance, supposing that there are [distinct kinds of] truths, does not make for a difference in its application. [...] As far as I can see, the same consideration applies to all the rules of natural deduction. The introduction and elimination rules do not appear to be sensitive to putative differences between [kinds of] truth, supposing there are such things.[...] Now if the distinction between the two sorts of truth postulated by the pluralist is inferentially irrelevant, truth pluralism has a serious problem. [...] It is difficult to believe that we need predicates corresponding to [kinds of] truth in addition to the unique inferentially relevant truth predicate we need and are lucky enough to have. $(385)$

Tappolet appears to be making a few main points here. First, she claims the rules of natural deduction are blind to different properties. Second, because of this, distinct truth properties cannot be distinguished inferentially. Third, these (completely general) rules of natural deduction can be characterized by a unique inferentially relevant truth property. Fourth, given these two claims, any other truth properties over and above the inferentially relevant one are superfluous.

I think that the strong pluralist can grant Tappolet's first claim. But surely it does not follow from this that truth properties cannot be distinguished inferentially. After all, an inferentialist who attempted to add a truth predicate for each truth property would want to characterize those by their own inferential rules. Natural deduction rules for the connectives

\footnotetext{
${ }^{9}$ However, see objection 1 in $\$ 2.4$ for related discussion.
} 
and quantifiers are not the only possible grounds for distinguishing kinds of truth. I see no reason for thinking that truth properties in different domains could not be distinguished inferentially. There might well be certain sorts of inferences which are (locally) valid in one domain, that are (locally) invalid in others. Take, for example, any epistemically constrained discourse (e.g. morality). In such a case, there is the valid inference from the truth of a proposition to knowability of that proposition. But this may not be valid for other truth properties in more realist domains (e.g. history or science).

Turning to Tappolet's third claim: what is this general truth property expressed by the 'unique inferentially relevant truth predicate' that we are 'lucky enough to have'? It had better not be, on pain of inconsistency, one characterized by the simple truth-in and truth-out rules $\sqrt{10}$ Such a truth predicate would have to be restricted to non-paradoxical sentences in order to function at all in the setting of classical logic. But one might take this as a compelling reason against the existence of general truth - as motivation for strong pluralism 11

I make these suggestions, however, only to dismiss them. Strong truth pluralists should not, merely by virtue of their strong pluralism, be committed to a proof-theoretic account of validity. Strong pluralism ought to be consistent with a semantic account of validity as well.

In the literature on mixed inferences, there are two other accounts of validity that are consistent with strong pluralism. Pedersen [30] responds to Tappolet by showing that horn (c) is not so unpalatable. He gives a plural (re)interpretation of validity: an argument is valid if and only if whenever the premises have a property that is among the truth properties, the conclusion must have a property that is among the truth properties. This strategy is promising in that it allows for valid mixed inferences to be 'truth-preserving' by interpreting truth plurally. Technical details aside, Pedersen's theory is held captive to the view that plural quantifiers are ontologically innocent. It may be that plural locutions like 'are among the truth properties' denote sets, or second-order properties, or the like. If so, Tappolet could object that we are smuggling in a general truth property after all, thereby falling prey to horn (b) of the trilemma.

\footnotetext{
${ }^{10}$ By truth-IN, I mean: $A \vdash T\langle A\rangle$. By truth-out, I mean: $T\langle A\rangle \vdash A$.

${ }^{11}$ See [11] for a more sophisticated defense of this line of argument. See also McGee [27] and Scharp [33. 34] who both conclude from the semantic paradoxes that we need two kinds of truth to do the job Tappolet thinks can be done by general truth.
} 
Debate over the innocence of plurals is beyond our scope here, and so we set this approach aside.

JC Beall [5] proceeds via a similar strategy. He has given a many-valued (re)interpretation of validity according to which truth properties are modeled by designated values, a standard notion in some non-classical logics. Designation, a purely model-theoretic notion, is preserved across valid inferences, not truth. Tappolet [40] has contended that, in effect, Beall is using designation as a general truth property.

It remains true that sentences which are $T_{1}$ and sentences which are $T_{2}$ share a common feature - they are designated. And it is difficult to believe this is not a kind of truth. (384)

Thus, Tappolet believes Beall's approach likewise fails to avoid horn (b) of the trilemma as well. I have previously argued (with Caret [8]), that designation is not a kind of truth. And so I think Beall's proposal is amenable to the strong pluralist. However, there are those who share Tappolet's suspicion that, if a single property is being preserved over all valid inferences then that property is really truth. So, to make things particularly difficult on the strong pluralist, let us set this approach aside as well.

If I have tracked the dialectic correctly, behind Tappolet's dilemma there is a truly difficult challenge to the strong pluralist.

Hard Problem of Mixed Inferences Give standard, non-revisionary, semantic account of the validity of mixed inferences that does not appeal (implicitly or explicitly) to any single property that is preserved.

This might appear to be an impossible task - that Tappolet has set herself up for a slam dunk. But I claim strong pluralists can meet the challenge. The key to meeting the challenge is an algebraic semantics, and a corresponding definition of validity.

\section{Validity for Strong Truth Pluralists}

To solve the Hard Problem of Mixed Inferences, I give a formal semantics together with an algebraic definition of validity that models the strong pluralist's view. I then show that validity 
in this formal language is classical. I discuss how the algebraic definition of validity sheds some philosophical light on Tappolet's dilemma.

\subsection{A Formal Semantics}

To model validity for strong pluralists, we need to first model the notion that each domain of discourse has a different truth property. To begin, our semantic values $V$ are ordered $n$-tuples, where $n$ is the number of domains. Each member of the tuple is either a 1 or a 0 .

$$
V=\left\{\left\langle a_{1}, \ldots, a_{n}\right\rangle \mid \text { each } a_{i} \in\{1,0\}\right\}
$$

If 1 appears in the $i$ th place, this represents that the proposition is in domain ${ }_{i}$ and is $\operatorname{true}_{i}$; 0 in the $i$ th place represents that the proposition is in domain ${ }_{i}$ and is false $e_{i}$. One should be careful not to think of the numeral 1 as being or even representing a truth property. It is merely a marker that represents that a proposition has the relevant truth property for its domain. In particular, one should not assume that the same numeral appearing in different places in the tuple means that the same truth property is had in different domains. (More on this possible confusion later, in §2.4)

Assume that atomic propositions have exactly one truth property (if they have any at all) ${ }^{12}$ So, our atomic values are given thus:

$$
V_{\alpha}=\left\{\left\langle a_{1}, \ldots, a_{n}\right\rangle \mid \exists ! i\left(a_{i}=1\right)\right\} \cup\langle 0, \ldots, 0\rangle
$$

Now we define valuation functions on the set of propositions. A valuation is admissible iff it satisfies the following:

1. For all atomic propositions $A, v(A) \in V_{\alpha}$.

2. For all propositions $A$, if $v(A)=\left\langle a_{1}, \ldots, a_{n}\right\rangle$ then $v(\neg A)=\left\langle 1-\left(a_{1}\right), \ldots, 1-\left(a_{n}\right)\right\rangle$.

\footnotetext{
${ }^{12}$ As noted earlier, this assumption may be dropped. Shapiro [36] has noted that in a first-order setting with a truth predicte (plus resources for diagonalization) the distinction between atomic and compound propositions is less than clear cut.
} 
3. For all propositions $A$ and $B$, if $v(A)=\left\langle a_{1}, \ldots, a_{n}\right\rangle$ and $v(B)=\left\langle b_{1}, \ldots, b_{n}\right\rangle$ then $v(A \vee B)=$ $\left\langle a_{1}+b_{1}, \ldots, a_{n}+b_{n}\right\rangle{ }^{13}$

4. For all propositions $A$ and $B$, if $v(A)=\left\langle a_{1}, \ldots, a_{n}\right\rangle$ and $v(B)=\left\langle b_{1}, \ldots, b_{n}\right\rangle$ then $v(A \wedge B)=$ $\left\langle a_{1} \times b_{1}, \ldots, a_{n} \times b_{n}\right\rangle$.

We can then define the other operators in the standard way: $A \supset B:=\neg A \vee B$, and $A \equiv B:=(A \supset$ $B) \wedge(B \supset A)$. A simple check reveals that these operations are just the classical operations on the component truth properties.

Now towards defining consequence; first define the algebraic order on our set of values:

Order $v(A) \leq v(B):=v(A \vee B)=v(B)$

It is easy enough to show that this is a partial order. For reflexivity, $v(A \vee A)=\left\langle a_{1}+a_{1}, \ldots, a_{n}+a_{n}\right\rangle=$ $\left\langle a_{1}, \ldots, a_{n}\right\rangle=v(A)$, since $1+1=1$ and $0+0=0$. For antisymmetry, assuming $v(A \vee B)=v(B)$ and $v(B \vee A)=v(A)$, then since $v(A \vee B)=v(B \vee A)$ we have it that $v(A)=v(B)$. For transitivity, assuming $v(A \vee B)=v(B)$ and $v(B \vee C)=v(C)$, we know that $v((A \vee B) \vee C)=v(C)$ by substitution. By associativity of $\vee$ and substitution, we have $v(A \vee C)=v(C)$.

Using this partial order, we can define logical consequence for strong pluralists thus:

Consequence $\mathcal{X}_{\vDash_{S P}} A$ iff $\bigwedge_{x \in \mathcal{X}} v(x) \leq v(A)$ for all admissible valuations $v$.

That is, an argument from $\mathcal{X}$ to $A$ is valid whenever the conjunction of the premises in $\mathcal{X}$ has a value less than (or equal to) the value of the conclusion.

\subsection{F Is Classical}

One might wonder what logic is given by $\vDash_{S P}$. The answer: classical propositional logic. Here is why.

First, on the given ordering $\leq$, the value of $(A \vee B)$ is just the least upper bound of the values of $A$ and $B$; likewise the value of $(A \wedge B)$ is just the greatest lower bound of the values of $A$ and $B$. To show that $v(A \vee B)$ is an upper bound of $v(A)$ and $v(B)$, we just show that $v(A) \leq v(A \vee B)$ and $v(B) \leq v(A \vee B)$. So, we just need to show that $v(A \vee(A \vee B)=v(A \vee B)$ which we have by

\footnotetext{
${ }^{13}$ Here $\mathrm{I}$ am assuming that $1+1=1$.
} 
associativity of $\vee$. (Likewise for $v(B \vee(A \vee B))$.) To show that $v(A \vee B)$ is the least upper bound, we show that for any upper bound $x$ of $v(A)$ and $v(B)$ s.t. $x \leq v(A \vee B), v(A \vee B) \leq x$. This follows from the associativity and idempotence of $\vee$. The same sort of argument can be given for $A \wedge B$ qua greatest lower bound of $v(A)$ and $v(B)$. Algebraically, our set of values with conjunction and disjunction is a lattice. Informally, this means that conjunction and disjunction behave nicely with respect to the order $\leq$ : the value of a conjunction is always less than (or equal to) the values of each of its conjuncts, and dually for disjunctions.

Second, I note that disjunction distributes over conjunction: $v(A \vee(B \wedge C))=v((A \vee B) \wedge$ $(A \vee C))$. We know that $v(A \vee(B \wedge C))=\left\langle a_{1}+\left(b_{1} \times c_{1}\right), \ldots, a_{n}+\left(b_{n} \times c_{n}\right)\right\rangle$ by the semantics for $\vee$ and $\wedge$. But arithmetic gives $a_{i}+\left(b_{i} \times c_{i}\right)=\left(a_{i}+b_{i}\right) \times\left(a_{i}+c_{i}\right)$ for each component $i$. But $\left\langle\left(a_{1}+b_{1}\right) \times\left(a_{1}+c_{1}\right), \ldots,\left(a_{n}+b_{n}\right) \times\left(a_{n}+c_{n}\right)\right\rangle$ just is the correct semantic value for $(A \vee B) \wedge(A \vee C)$. Algebraically, we have a distributive lattice. Informally, this guarantees that conjunctions and disjunctions behave nicely with each other.

Third, the value $\langle 1,1, \ldots, 1\rangle=\top$ is the top value on the given ordering; that is, all other values $\left\langle a_{1}, \ldots, a_{n}\right\rangle \leq T$. Likewise, $\langle 0,0, \ldots, 0\rangle=\perp$ is the bottom value. Notice, too that since $0 \times 1=0$ and $0+1=1$, we have it that $a_{i}+\left(1-a_{i}\right)=1$ and $a_{i} \times\left(1-a_{i}\right)=0$ for each $a_{i}$. So, $v(A \vee$ $\neg A)=\langle 1,1, \ldots, 1\rangle=\top$, and $v(A \wedge \neg A)=\langle 0,0, \ldots, 0\rangle=\perp$ for all $A$. Algebraically, this structure is called a complemented distributive lattice. So, negation behaves nicely with conjunction and disjunction: we have typical de Morgan equivalences, ExCluded Middle, and ex falso QUODLIBET. It also ensures the validity of the double negation laws.

These facts constitute a proof that $\langle V, \vee, \wedge, \neg, \top, \perp\rangle$ is a Boolean Algebra, since all complemented distributive lattices are Boolean algebras. That means that $\vDash_{S P}$ as defined above is just the consequence relation of classical propositional logic. In the standard semantics of classical logic, the Boolean algebra simply consists of the values $T$ and $\perp$ with the usual operations. But any Boolean algebra suffices, including the one given by the formal semantics above.

\subsection{Solving the Problem}

Strong pluralists can now deliver an account of validity that satisfies Tappolet's demands. Recall that the Hard Problem of Mixed Inferences was to give standard, non-revisionary, se- 
mantic account of validity that does not appeal (implicitly or explicitly) to any single property that is preserved.

Algebraic conceptions of validity are a completely standard idea. Algebraic semantics are perhaps some of the most useful methods in the study of logic, and have led to countless developments in its history. Algebraic conceptions of logical consequence are at least as old as semantics for classical propositional logic itself - indeed, Boolean algebras were developed for precisely this purpose. Of the three dominant approaches to logical consequence - prooftheoretic, algebraic, and model-theoretic - the youngest is model theory.

The fact that $\vDash_{S P}$ is extensionally equivalent to the classical truth-preservation account should serve to alleviate the thought that this notion of validity is somehow revisionary. Monists and pluralists can agree: the valid inferences are left untouched.

The algebraic definition of validity is likewise a thoroughly semantic idea. Propositions are still assigned values; it is just that they are not monistic values any more; they are $n$-tuples representing many truth properties. Compound propositions are semantically functional - they depend entirely on the values of their constituent parts. Moreover, the meanings of the logical connectives can be seen as being given by their operations on values. Contrary to the monist, however, the strong pluralist now claims that these operations are operations on components of values - the various truth properties - and not primarily on the values themselves.

Finally, the notion of order, on which the algebraic conception of validity depends, does not appeal to any single truth property being preserved. After all, a semantic value $\left\langle a_{1}, \ldots, a_{n}\right\rangle$ is 'less than' another $\left\langle b_{1}, \ldots, b_{n}\right\rangle$ just in case $a_{i} \leq b_{i}$ for each domain $i$. Semantically speaking, a value is less than (or equal to) another just if the latter includes all the truth properties of the former. No single truth property plays a privileged role; every truth property is equally involved in the definition of validity.

It appears the Hard Problem of Mixed Inferences can be solved. Before claiming victory, however, allow me to address two further concerns. 


\section{4 ОвJECTIONS AND REPLIES}

Objection 1: Given that the same numeral (e.g. 1) appears in different places of the tuple, it appears you are smuggling in a general truth property after all. The numeral 1 represents that 'a proposition has the relevant truth property for its domain', which appears to introduce some second-order property or the like. Thus, the proposal suffers from the same problem that Beall's and Pedersen's do - an implicit reliance on a general truth property of some sort ${ }^{14}$

Reply: Let us call this the 'same component, same property' objection. As mentioned above, one should be careful not to read too much metaphysics into the formal machinery. Luckily, one could just as easily have chosen distinct markers to represent the truth property in distinct domains.

$1 \mathrm{~s}$ and 0 s are used for notational convenience; they are the standard names for the elements in the simplest (two-element) Boolean algebra. Fortunately, elements from any Boolean algebra will suffice for component values within a domain. Starting with distinct Boolean algebras, $A_{1}, A_{2}, A_{3}, \ldots$, simply choose an element from $A_{1}$ as the first component, an element from $A_{2}$ as the second component, etc. All that is required is that each distinct algebra $A_{i}$ with its distinct ordering $\leq_{i}$ has a top element based on that ordering. And that is, of course, guaranteed by the algebra's being Boolean.

One might suspect that 'being the top element of a Boolean algebra' is somehow playing a general truth role. But the ordering relation $\leq_{i}$ on individual algebras is defined entirely within a domain. Indeed, the domain-relative ordering relation is completely determined by the behavior of disjunction within that domain: $a_{i} \leq_{i} b_{i}$ if and only if $a_{i}+b_{i}=b_{i}$. Strong pluralists face no (new) problems giving semantics for unmixed conjunctions; this only requires the domain-specific truth properties. But then the rest of the semantics falls out immediately. In short, the the definition of validity given above can be achieved without ever having a component appear in more than one place in the tuple, without ever explicitly or implicitly invoking a general truth property.

\footnotetext{
${ }^{14}$ Thanks to Doug Edwards for pressing on me the importance of responding to this sort of objection.
} 
Objection 2: The ordering relation on which validity is defined models the idea that all truth properties are preserved throughout an inference. This seems to conflict with the initial motivation for giving an account of the validity of mixed inferences. After all, consider two true atomic propositions from different domains. Let $v(p)=\langle 1,0\rangle$ and $v(q)=\langle 0,1\rangle$. Their conjunction is false in every domain: $v(p \wedge q)=\langle 0,0\rangle$. But then, apparently one can validly infer any proposition at all from $p$ and $q$ !

Reply: Fortunately, the problem relies on a mistake: the objector is considering only a single valuation. One must consider all possible valuations of $p$ and $q$, and on some evaluations, these sentences may be interpreted as having the same semantic value (and hence having the same truth properties). Such valuations serve as potential counterexamples to the argument from $p$ and $q$ to an arbitrary proposition. 15

It might seem that interpreting atomic sentences from different domains in this way goes against the spirit of truth pluralism; that somehow all possible valuations of sentences ought to respect the fact that they have different content which decidedly places them in different domains. On the contrary, logical consequence is a formal notion. Validity in formal logic is independent of content, and is in an important sense topic neutral. Pluralists (even strong pluralists) are not barred from thinking that valid inference depends only on the logical form of an argument, and not on the content of the particular premises of an instance of an argument form. Even when making domains of discourse and their corresponding truth properties explicit in a definition of validity, this level of abstraction is available, and perhaps desirable, as an account of formal inference.

Does this compromise the response to the Hard Problem of Mixed Inferences? Not at all. What these considerations show is that arguments with the right sort of formal structure are valid regardless of whether the premises are interpreted as being from the same domain or entirely different domains. Whether an inference is 'mixed' or not has no effect on the question of its validity. And the strong pluralist can achieve this without any explicit or implicit appeal to a single general truth property, relying only on the many domain-specific truth properties.

\footnotetext{
15Thanks to Dave Ripley for discussion here.
} 
I conclude that the strong pluralist can solve the Hard Problem of Mixed Inferences.

\section{Extending the IDEA}

I have shown that the above consequence relation is entirely coextensive with classical logic. There are those for whom this would be an unwelcome result. Indeed, some truth pluralists have endorsed the view that, not only truth, but also valid inference varies across domains of discourse. Lynch $[25,26]$ has recently suggested that truth pluralism has a natural fit with a certain kind of logical pluralism. ${ }^{16}$ Moreover, Wright [42] has suggested that the correct account of truth in epistemically constrained domains might be something like superassertability, which sits most naturally within intuitionistic logic. In this section, I extend the algebraic account of validity to non-classical domains, showing how the account can handle domains for which paracomplete, paraconsistent, and intuitionistic logic seem most appropriate.

\subsection{Many-Valued Domains}

In the classical semantics given above, it was assumed that for every truth-apt proposition $p$ and every domain $i, p$ is either true ${ }_{i}$ or false $e_{i}$. That is, if a proposition does not have the truth property for a given domain, then it must have the falsity property for that domain. Moreover,

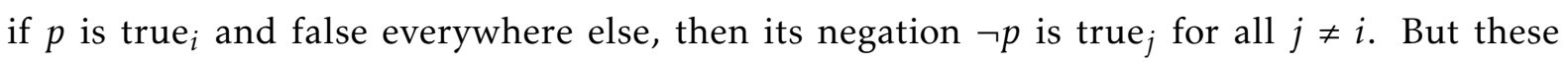
assumptions might well be questioned. For example, why think that the negation of a true descriptive proposition must be morally true, mathematically true, etc.?

To alleviate these concerns, one might move to a non-classical logic. ${ }^{17}$ Let us introduce a third component, $\frac{1}{2}$, such that a $\frac{1}{2}$ appearing in the $i$ th place of a semantic value represents that a proposition is undefined in $\operatorname{domain}_{i}$. Semantic values, then, are $n$-tuples of these three values.

$$
V^{*}=\left\{\left\langle a_{1}, \ldots, a_{n}\right\rangle \mid \text { each } a_{i} \in\left\{1, \frac{1}{2}, 0\right\}\right\}
$$

\footnotetext{
${ }^{16}$ This logical pluralism is domain-relative, and as such distinct from the view endorsed by Restall and Beall [7].

17But see Azzouni and Bueno [2], and Greenough [18] for recent attempts to accommodate 'indeterminacy' within a classical framework.
} 
It has been assumed throughout that atomic propositions are members of exactly one domain ${ }^{18}$ So, atomic values will be undefined in every place but one.

$$
V_{\alpha}^{*}=\left\{\left\langle a_{1}, \ldots, a_{n}\right\rangle \mid \exists ! i\left(a_{i} \in\{1,0\}, a_{j}=\frac{1}{2} \text { where } j \neq i\right)\right\}
$$

The semantic clauses for the connectives are analogous to the classical case: on the standard (numerical) ordering of $1, \frac{1}{2}$, and 0 , the value of a conjunction will be the greatest lower bound of the values of its conjuncts; the value of a disjunction is the least upper bound of the values of its disjuncts. It is worth noting that atomic propositions and their negations always only have the truth (or falsity) property for their domain. If $v(p)=\left\langle\frac{1}{2}, \ldots, 1, \ldots, \frac{1}{2}\right\rangle$, then $v(\neg p)=$ $\left\langle 1-\frac{1}{2}, \ldots, 1-1, \ldots, 1-\frac{1}{2}\right\rangle=\left\langle\frac{1}{2}, \ldots, 0, \ldots, \frac{1}{2}\right\rangle$.

Consequence for this logic is treated in the same algebraic way:

Consequence $\mathcal{X} \vDash_{S_{3}} A$ iff $\bigwedge_{x \in \mathcal{X}} v(x) \leq v(A)$ for all admissible valuations $v$.

What logic corresponds to $\vDash_{S_{3}}$ ? Notice, one can allow cases for which $v(A \vee \neg A) \neq \mathrm{T}$. For example, let $v(A)=\left\langle\frac{1}{2}, \ldots, \frac{1}{2}\right\rangle$. But that is just the semantic value given by $v(\neg A)$ too. Hence $v(A \vee \neg A)=\left\langle\frac{1}{2}, \ldots, \frac{1}{2}\right\rangle$. As a result of this fact, we do not have the validity of the LAw OF EXCLUDED MIDDLE. So, $\vDash_{S_{3}}$ is a paracomplete logic. Moreover, similar reasoning can be used to show that $v(A \wedge \neg A)=\left\langle\frac{1}{2}, \ldots, \frac{1}{2}\right\rangle$, for some $A$. Hence, we do not have the validity of Ex FALso QuOdLIBEt. So, $\vDash_{S_{3}}$ is paraconsistent as well. In fact, this semantics gives a weak de Morgan logic - the same logic that results from adding the inference $A \wedge \neg A \vdash B \vee \neg B$ to Dunn's [1] First-Degree Entailment. Alternatively, it is the intersection of Strong Kleene [20] and Priest's [32] Logic of Paradox 19

The fact that the semantic values, semantic clauses, and definition of validity in $\vDash_{S P}$ and $F_{S_{3}}$ are identical has a nice formal consequence. One can, if one wishes, insist that propositions in some domains are always classically evaluated while allowing for non-classical domains of discourse. One merely stipulates that, at some coordinates in the $n$-tuples, components are always only selected from 1 and 0 . The result, then, is that a certain sort of logical pluralism,

\footnotetext{
${ }^{18}$ Again, for pluralists who accept that an atomic proposition may be a member of more than one domain, this assumption may be dropped without difficulty.

${ }^{19}$ The name $S_{3}$, due to Hartry Field [17] p. 81, is for 'Symmetric Kleene Logic'. See also Kremer [22].
} 
in addition to truth pluralism, can be handled in a strong pluralist way.

\subsection{INTUITIONISM}

Paraconsistent and paracomplete logics are not standardly endorsed by pluralists $\stackrel{20}{0}$ However, recently Lynch [25, 26], following an earlier suggestion by Wright [42], has suggested that intuitionistic logic is the appropriate logic for domains of discourse that are epistemically constrained. It is worth showing, then, that the algebraic definition of validity is amenable to intuitionistic logic being the correct logic for some domains of discourse.

To begin, we need to explain the notion of a Heyting algebra. Heyting algebras are generalizations of Boolean algebras. But instead of being complemented as in $\$ 2.2$, they are pseudocomplemented. Formally, $\langle H,+, \times, \Rightarrow, 1,0\rangle$ is a Heyting algebra iff:

- $\langle H,+, \times, 1,0\rangle$ is a bounded distributive lattice; and

- for every $a, b \in H$ there is a greatest element $x \in H$ such that $a \times x \leq c$ (this element is the relative pseudo-complement and is denoted $a \Rightarrow b$ ).

In Heyting algebras (and intuitionistic logic generally), negation is defined: if $v(A)=a$ then $v(\neg A):=a \Rightarrow 0$.

Now, toward defining our set of semantic values, let $H_{1}, \ldots, H_{n}$ be arbitrary Heyting algebras, one for each 'domain'. Now, semantic values will be $n$-tuples such that at each $i$ th place is a member of the Heyting algebra $H_{i}$.

$$
V^{\prime}=\left\{\left\langle a_{1}, \ldots, a_{n}\right\rangle \mid \text { each } a_{i} \in H_{i}\right\}
$$

As in the semantics prior, 1 in the $i$ th place represents that the proposition is in domain ${ }_{i}$ and has the property true ${ }_{i}$. Likewise for 0 in the $i$ th place and false $e_{i}$. Atomic values are such that there is a 1 or 0 in a single coordinate.

$$
V_{\alpha}^{\prime}=\left\{\left\langle a_{1}, \ldots, a_{n}\right\rangle \mid \exists ! i\left(a_{i} \in\{1,0\}, a_{j} \in H_{j} \text { where } j \neq i\right)\right\}
$$

\footnotetext{
${ }^{20}$ In early drafts, Lynch [26] experimented with using Strong Kleene as the logic for some domains, eventually siding with intuitionistic logic.
} 
The semantics for our connectives are defined similarly as the classical case: conjunctions are component-wise greatest lower bounds; disjunctions are component-wise least upper bounds. The only difference is addition of a binary conditional operator, and a revised negation clause:

5. For all propositions $A$ and $B$, if $v(A)=\left\langle a_{1}, \ldots, a_{n}\right\rangle$ and $v(B)=\left\langle b_{1}, \ldots, b_{n}\right\rangle$ then $v(A \rightarrow B)=$ $\left\langle a_{1} \Rightarrow b_{1}, \ldots, a_{n} \Rightarrow b_{n}\right\rangle$.

2'. For all propositions $A$, if $v(A)=\left\langle a_{1}, \ldots, a_{n}\right\rangle$, then $v(\neg A)=\left\langle a_{1} \Rightarrow 0, \ldots, a_{n} \Rightarrow 0\right\rangle$.

Consequence $\vDash_{I}$ can be defined in the standard algebraic way.

Defining values and connectives in this way is sufficient for $\left\langle V^{\prime}, \vee, \wedge, \rightarrow, \top, \perp\right\rangle$ itself to be a Heyting algebra. ${ }^{21}$ The fact that admissible valuations consider arbitrary Heyting algebras $H_{1}, \ldots, H_{n}$ as our basis gives us the result that $\vDash_{I}$ is equivalent to intuitionistic logic.

Finally, because all Boolean algebras are Heyting algebras, our choice of any $H_{i}$ could well have been Boolean. This is consistent with the idea that, even if intuitionistic logic is appropriate for reasoning within some domains, classical logic might still be maintained as the correct logic for reasoning within other domains.

To conclude, the Hard Problem of Mixed Inferences can be solved to Tappolet's specifications. Strong pluralists about truth do not need to appeal to any single general truth property in order to have a standard, non-revisionary, semantic account of validity. Moreover, they can allow that the logic of unmixed inferences can sometimes be domain-dependent. There is no barrier to extending this strong pluralist solution to a logical pluralism 22

\section{REFERENCES}

[1] N.D. Belnap A.R. Anderson and J. M. Dunn. Entailment: The Logic of Relevance and Necessity, volume 2. Princeton University Press, 1992.

[2] J. Azzouni and O. Bueno. On what it takes for there to be no fact of the matter. Noûs, 42(4):753-769, 2008.

\footnotetext{
${ }^{21}$ Heyting algebras are varieties. Every variety is closed under direct products. Thanks to Dave Ripley and Jeff Russell for discussion here.

${ }^{22}$ Thanks to JC Beall, Doug Edwards, Michael Hughes, Michael Lynch, Dave Ripley, Jeff Russell, Paula Sweeney, Crispin Wright for comments and discussion on earlier versions of this paper. Additional thanks to an anonymous referee whose comments led to a number of improvements.
} 
[3] R. Barnard and T. Horgan. Truth as mediated correspondence. The Monist, 89(1):28-49, 2006.

[4] R. Barnard and T. Horgan. The synthetic unity of truth. In N.J. Pedersen and C.D. Wright, editors, Truth and Pluralism: Current Debates. Oxford University Press, forthcoming.

[5] JC Beall. On mixed inferences and pluralism about truth predicates. The Philosophical Quarterly, 50:380-382, 2000.

[6] JC Beall. Deflated truth pluralism. In N.J. Pedersen and C.D. Wright, editors, Truth and Pluralism: Current Debates. Oxford University Press, forthcoming.

[7] JC Beall and G. Restall. Logical Pluralism. Oxford University Press, Oxford, 2006.

[8] C. Caret and Aaron J. Cotnoir. True, false, paranormal, and 'designated'?: a reply to Jenkins. Analysis, 68.3, July 2008.

[9] A. J. Cotnoir. A pluralist account of satisfaction. ms.

[10] A. J. Cotnoir. Generic truth and mixed conjunctions: Some alternatives. Analysis, $69(3): 473-479,2009$.

[11] A. J. Cotnoir. Pluralism and paradox. In N.J. Pedersen and C.D. Wright, editors, Truth and Pluralism: Current Debates. Oxford University Press, forthcoming.

[12] D. Dorsey. A coherence theory of truth in ethics. Philosophical Studies, 127:493-523, 2006.

[13] D. Dorsey. Truth and error in morality. In N. Pedersen and C.D. Wright, editors, New Waves in Truth. Palgrave Macmillan, 2010.

[14] D. Edwards. How to solve the problem of mixed conjunctions. Analysis, 68.2:143-149, April 2008.

[15] D. Edwards. Truth conditions and truth: Resolving the problem of mixed conjunctions. Analysis, 69.4:684-688, 2009. 
[16] D. Edwards. Truth, winning, and simple determination pluralism. In N.J. Pedersen and C.D. Wright, editors, Truth and Pluralism: Current Debates. Oxford University Press, forthcoming.

[17] H. Field. Saving Truth From Paradox. Oxford University Press, 2008.

[18] P. Greenough. Indeterminate truth. In P. French, editor, Truth and Its Deformities. Midwest Studies in Philosophy, 2008.

[19] T. Horgan. Contextual semantics and metaphysical realism: Truth as indirect correspondence. In M.P. Lynch, editor, The Nature of Truth. MIT Press, Cambridge, MA, 2001.

[20] S.C. Kleene. Introduction to Metamathematics. North Holland, 1952.

[21] M. Kölbel. 'true' as ambiguous. Philosophy and Phenomenological Research, 77:359-384, 2008.

[22] P. Kremer. Kripke and the logic of truth. Journal of Philosophical Logic, 17:225-278, 1988.

[23] M.P. Lynch. A functionalist theory of truth. In M.P. Lynch, editor, The Nature of Truth, pages 723-750. MIT Press, Cambridge, MA, 2001.

[24] M.P. Lynch. Truth and multiple realizability. Australasian Journal of Philosophy, 82(3):384408,2004 .

[25] M.P. Lynch. Alethic pluralism, logical consequence and the universality of reason. Midwest Studies in Philosophy, 32:122-140, 2008.

[26] M.P. Lynch. Truth as One and Many. Oxford University Press, Oxford, 2008.

[27] V. McGee. Truth, Vagueness, and Paradox: An Essay on the Logic of Truth. Hackett, 1991.

[28] V. McGee. Two conceptions of truth? Philosophical Studies, 124:71-104, 2005.

[29] N. J. Pedersen and C.D. Wright. Varieties of alethic pluralism (and why alethic disjunctivism is relatively compelling). In N.J. Pedersen and C.D. Wright, editors, Truth Pluralism: Current Debates. Oxford University Press. forthcoming. 
[30] N.J. Pedersen. What can the problem of mixed inferences teach us about pluralism? The Monist, 89(1):102-117, 2006.

[31] N.J. Pedersen. Stabilizing alethic pluralism. The Philosophical Quarterly, 60:92-108, 2010. forthcoming.

[32] G. Priest. The logic of paradox. Journal of Philosophical Logic, 8(4):219-241, 1979.

[33] K. Scharp. Alethic vengeance. In J. C. Beall, editor, Revenge of the Liar: New Essays on the Paradox, pages 272-319. Oxford University Press, 2007.

[34] K. Scharp. Replacing truth. Inquiry, 50(6):606-621, 2007.

[35] S. Shapiro. Review of Truth as One and Many. Notre Dame Philsophical Reviews, 09.22.2009, 2009 .

[36] S. Shapiro. Truth, function, and paradox. Analysis, forthcoming.

[37] G. Sher. In search of a substantive theory of truth. The Journal of Philosophy, 101:5-36, 2004 .

[38] G. Sher. Functional pluralism. Philosophical Books, 46(4):311-330, 2005.

[39] C. Tappolet. Mixed inferences: a problem for pluralism about truth predicates. Analysis, 57:209-210, 1997 .

[40] C. Tappolet. Truth pluralism and many-valued logics: a reply to Beall. The Philosophical Quarterly, 50:382-385, 2000.

[41] T. Williamson. A critical study of 'Truth and Objectivity'. International Journal of Philosophical Studies, 30:130-144, 1994.

[42] C. Wright. Truth and Objectivity. Harvard University Press, Cambridge, MA, 1992.

[43] C. Wright. Minimalism, deflationism, pragmatism, pluralism. In M.P. Lynch, editor, The Nature of Truth. MIT Press, Cambridge, MA, 2001. 
[44] C. Wright. Varieties of alethic pluralism. In N.J. Pedersen and C.D. Wright, editors, Truth and Pluralism: Current Debates. Oxford University Press, forthcoming.

[45] C.D. Wright. Is pluralism about truth inherently unstable? Philosophical Studies. forthcoming.

[46] C.D. Wright. On the functionalization of pluralist approaches to truth. Synthese, 145:128,2005 . 